\title{
SARCOPTERYGII DO EOPERMIANO DA BACIA DO PARANÁ, ESTADO DE SÃO PAULO
}

Artur CHAHUD

Setembrino PETRI

\begin{abstract}
RESUMO
Esta contribuição constitui uma revisão dos Sarcopterygii (Coelacanthimorpha - celacantos; Tetrapodomorpha - Osteolepimorpha e tetrápodes) do Eopermiano da Bacia do Paraná no Estado de São Paulo. O outro grupo importante de sarcopterígeos Dipnoi (peixes pulmonados) - ainda não foi registrado nesse estado no Eopermiano. Os Coelacanthimorpha foram observados no Grupo Itararé e nas formações Tatuí e Irati. Os arenitos e conglomerados da base do Membro Taquaral da Formação Irati são ricos em ictiofósseis, incluindo dentes labirintodontes atribuídos a Tetrapodomorpha, que variam de poucos milímetros a alguns centímetros, sendo, portanto, os maiores dentes já encontrados no Permiano em São Paulo. A fácies folhelho síltico do Membro Taquaral é a que apresenta maior abundância em número de escamas de Coelacanthimorpha, com espécimes de alguns centímetros de comprimento porém, até agora, nenhum outro Sarcopterygii foi identificado. Apesar dos celacantídeos atualmente serem indicadores de ambiente marinho profundo, no Paleozoico eles podem ser encontrados em rochas depositadas em ambientes de salinidades variadas e na água doce. Os Tetrapodomorpha também possuíam espécies que habitavam ambientes de salinidade variada e continentais. Para análise paleoambiental destes grupos é necessário o conhecimento do contexto sedimentar e da paleobiota associada.
\end{abstract}

Palavras-chave: Eopermiano, Coelacanthimorpha, Tetrapodomorpha, Bacia do Paraná.

\section{ABSTRACT}

This contribution comprises a review of Early Permian Sarcopterygii (Coelacanthimorpha - coelacanths; Tetrapodomorpha - Osteolepimorpha and tetrapods) of the Paraná Basin in the state of São Paulo. The other important group of sarcopterygians - Dipnoi (lungfishes) - have still not been recorded in São Paulo in the Early Permian. The Coelacanthimorpha have been reported in the Itararé Group, Tatuí and Irati formations. The sandstone and conglomerate at the base of the Taquaral Member of the Irati Formation are rich in icthyofossils, including labyrinthodont teeth that have been assigned to the Tetrapodomorpha. These teeth range from a few millimeters to a few centimeters in length, which makes them the largest teeth in the Permian of the State of São Paulo. The most frequent fish remains in the silty shale facies of the Taquaral Member are scales of Coelacanthimorpha, which may reach a few centimeters in size. Currently the coelacanths live in deep marine environments. However, during the Paleozoic they lived in waters of different salinities as well as in fresh waters. The Tetrapodomorpha were also able to live in waters of varied salinities and in continental settings. The paleoenvironmental analysis of ancient deposits with these groups of fossils thus requires a thorough survey of their sedimentary context as well as the associated paleobiota.

Keywords: Early Permian, Coelacanthimorpha, Tetrapodomorpha, Paraná Basin. 


\section{INTRODUÇÃO}

Na Era Paleozoica, os Sarcopterygii possuíam uma diversidade de formas e habitats. Atualmente compreendem os táxons Coelacanthimorpha (celacantos), Dipnoi (Peixes pulmonados) e Tetrapodomorpha (Tetrápodes e Osteolepimorpha) (AHLBERG \& JOHANSON 1998).

O Eopermiano da Bacia do Paraná em São Paulo inclui a parte superior do Grupo Itararé, Formação Tatuí (equivalente do Grupo Guatá na Região Sul) e a Formação Irati (membros Taquaral e Assistência). O Grupo Itararé, de idade permocarbonífera, possui poucas ocorrências de vertebrados e todas tiveram idades estipuladas como neocarboníferas. A Formação Tatuí é constituída por siltitos e arenitos finos bem selecionados, com baixíssima quantidade de fósseis em ocorrências localizadas, representados por microfósseis, lenhos, espículas de esponja, crustáceos e dentes e escamas de Palaeonisciformes (FÚLFARO et al. 1984, SILVA-SANTOS 1991, MEZZALIRA \& MARTINS NETO 1992). Na região centro-leste do Estado de São Paulo, em discordância sobre a Formação Tatuí, ocorrem arenitos conglomeráticos ou conglomerados pertencentes à base do Membro Taquaral (HACHIRO 1996, ASSINE et al. 2003, CHAHUD 2011, CHAHUD et al. 2012), ricos em ictiofósseis de Chondrichthyes e Osteichthyes (RAGONHA 1978; CHAHUD \& PETRI 2008a, b; 2009a, b; 2010b; CHAHUD et al. 2010). Acima desta camada ocorre fácies de folhelhos sílticos, predominante no Membro Taquaral, com fósseis dispersos de crustáceos e ictiofósseis e abundante registro de escamas de vertebrados (CHAHUD \& PETRI 2013a, b).

Esta contribuição revisa os fósseis atribuídos ao Grupo Sarcopterygii (Coelacanthimorpha - celacantos; Tetrapodomorpha - Osteolepimorpha e tetrápodes) do Eopermiano da Bacia do Paraná no Estado de São Paulo, considerando os tipos de preservação, diferenças litológicas e paleoambientes associados. Os Dipnoi (peixes pulmonados) ainda não foram registrados nesse estado no Eopermiano.

\section{COELACANTHIMORPHA}

Os Coelacanthimorpha (ou Actinistia) neopaleozoicos do Brasil são representados por escamas, pouco descritas e ilustradas na literatura, embora ocorram em diversas unidades litoestratigráficas. Fósseis articulados e bem preservados de celacantos ocorrem somente no Jurássico da Bacia do Parnaíba, Formação Pastos Bons (gênero Parnaibaia,
YABUMOTO 2008) e no Cretáceo da Chapada do Araripe, Formação Santana (Axelrodichthys e Mawsonia, MAISEY 1991), e da Bacia do Grajaú, Formação Codó (Axelrodichthys, CARVALHO \& MAISEY 2008).

Na Bacia do Paraná, BRYANT (1929) foi o pioneiro a descrever uma escama de celacantídeo proveniente de uma rocha descrita como tilito do Grupo Itararé (Estado de São Paulo). A segunda identificação, registrada por BARCELLOS (1975), também é proveniente do Grupo Itararé (Fácies Budó), Estado do Rio Grande do Sul. A autora descreveu diversas formas pertencentes a uma única espécie. RICHTER (1985) descreve uma escama coletada no topo da Formação Irati no Estado do Rio Grande do Sul. WÜRDIG -MACIEL (1975) e MARANHÃO (1995) mencionaram a presença de escamas na Formação Corumbataí, região de Piracicaba, Estado de São Paulo.

As escamas de Coelacanthimorpha encontradas nas formações Tatuí e Irati (CHAHUD \& PETRI 2009b; 2013a, b) possuem linhas de crescimento delgadas, retas, separadas por pequenos espaços ou unidas. Nas regiões mais distais da escama (em relação a zona de articulação), podem ser divergentes e interrompidas (Figura 1A), curvadas, fechadas ou concêntricas, de forma arredondada (Figura 1B; 1C; 2A e 2B) ou irregular. A espessura das linhas de crescimento atinge, no máximo, $0,01 \mathrm{~mm}$.

As escamas de Coelacanthimorpha tem comprimento e largura que variam de $5 \mathrm{~mm}$ a $10 \mathrm{~mm}$ e são muito delgadas; nos exemplares maiores, não ultrapassam $0,1 \mathrm{~mm}$ de espessura (medida sempre na zona de articulação).

Ossos e possíveis dentes também são encontrados nas formações Tatuí e Irati; pelo fato desses dentes serem muito semelhantes aos dos paleoniscoides (RICHTER et al. 1985, CHAHUD \& PETRI 2008b), sua identificação é incerta.

\section{TETRAPODOMORPHA}

Além dos Coelacanthimorpha, os Sarcopterygii do Eopermiano da Bacia do Paraná incluem os Tetrapodomorpha (Osteolepimorpha e tetrápodes). Os fósseis mais comuns são dentes e ossos isolados (RAGONHA 1978; CHAHUD \& PETRI 2009b, 2010a), ocasionalmente, escamas (DIAS \& RICHTER 2002) ou indivíduos articulados (BARBERENA 1998, DIAS \& BARBERENA 2001, DIAS \& SCHULTZ 2003). A dentição, tanto para os Osteolepimorpha como para os tetrápodes basais, é do tipo labirintodonte (grandes dentes de 

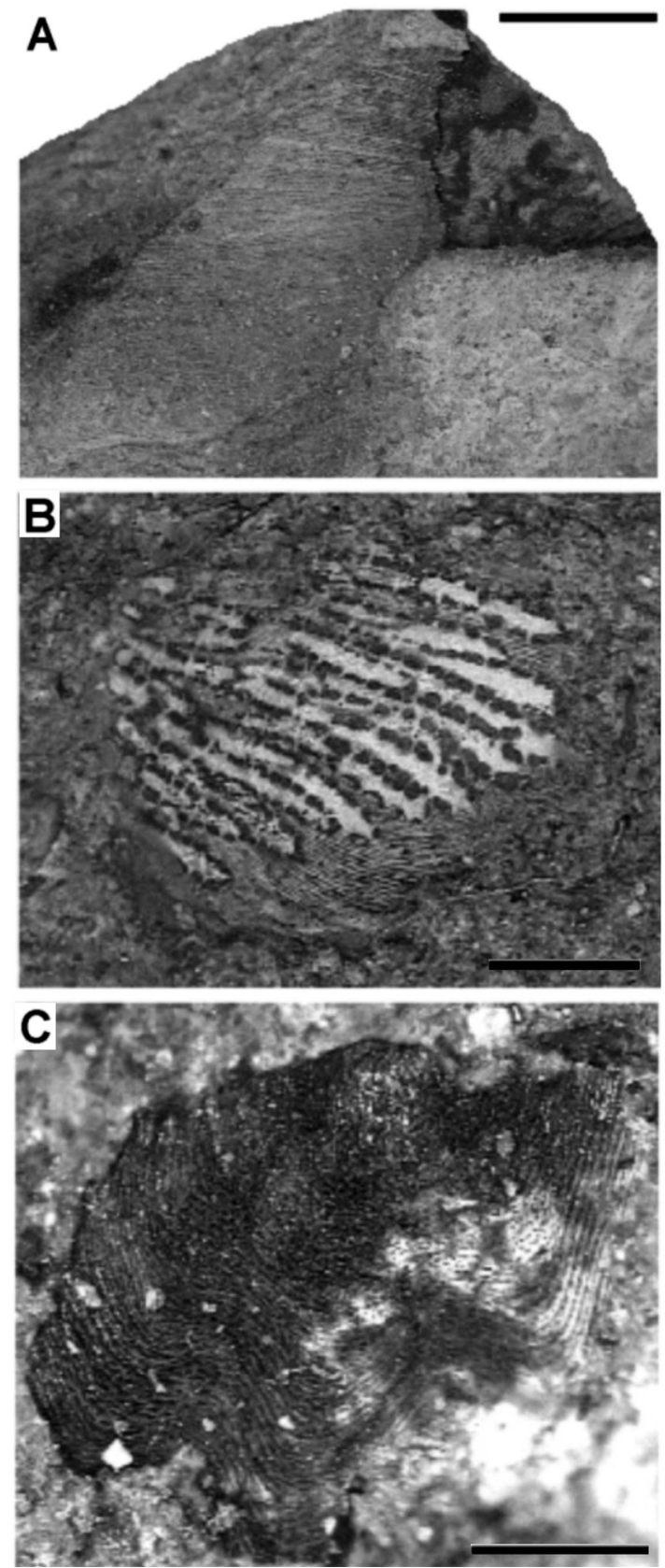

FIGURA 1 - Escamas fragmentadas de Coelacanthimorpha da região de Rio Claro, Estado de São Paulo (CHAHUD \& PETRI 2009b). A) Exemplar da Formação Tatuí (GP/2E-5965). Escala $2 \mathrm{~mm}$. B) Exemplar 1 da base arenosa do Membro Taquaral (GP/2E-5966). Escala 1mm. C) Exemplar 2 da base arenosa do Membro Taquaral (GP/2E-5967). Escala 0,6 mm.
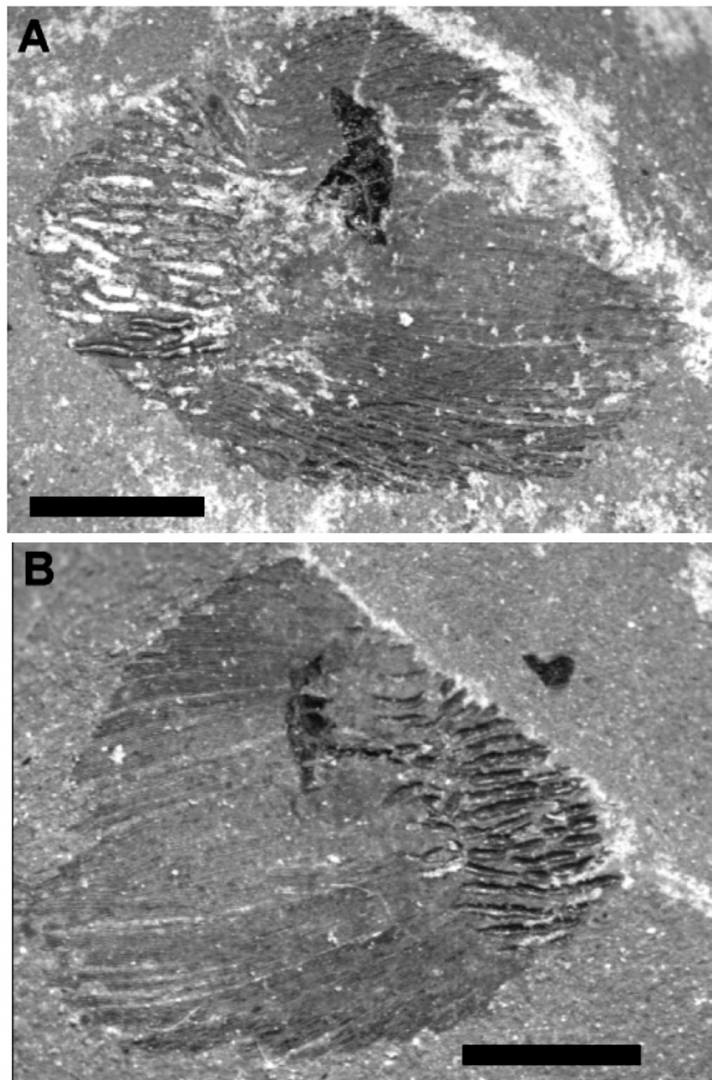

FIGURA 2 - Escama de Coelacanthimorpha do folhelho síltico do Membro Taquaral, Formação Irati, da região de Rio Claro (CHAHUD \& PETRI 2013a, b). A) Parte (GP/2E-5969a); B) Contraparte (GP/2E5969b). Escala $2 \mathrm{~mm}$.

formato cônico com dentina em dobras complexas na cavidade pulpar), o que dificulta a diferenciação desses grupos. Externamente, os dentes apresentam inúmeras estrias longitudinais próximas à base que, aos poucos, diminuem até desaparecerem completamente, próximo do topo.

Formas como o exemplar GP/2E-6309 (Figura 3A), da base da Formação Irati (CHAHUD \& PETRI 2009b), são de difícil classificação. O dente é fortemente estriado na parte inferior, possui uma cúspide arredondada por forte abrasão, forma cônica robusta, ligeiramente encurvada e exibe algumas fraturas ou rachaduras superficiais. Outros indícios, como a associação com possíveis ossos apendiculares (Figura 3C), também foram encontrados e a possibilidade de pequenos tetrápodes ou Osteolepimorpha não deve ser descartada.

O grupo Temnospondyli é o mais abundante dos tetrápodes no registro paleozoico, representados por grandes animais de hábitos aquáticos e terres- 
tres. Esse grupo habitou todos os continentes e duas superfamílias são encontradas no Permiano brasileiro: Archegosauroidea, de idade permo-carbonífera, e Rhinesuchoidea, permo-triássica (PRICE 1948, PUTZER 1954, BARBERENA 1972, COX \& HUTCHINSON 1991, BARBERENA 1998, DIAS 2000, DIAS \& BARBERENA 2001, DIAS \& SCHULTZ 2003, CISNEROS et al. 2005, ABREU \& DIAS-DA-SILVA 2006, CHAHUD \& PETRI 2010a, ELTINK \& DIAS 2012).

O dente GP/2T-202 (Fig. 3B) pertence, até o momento, ao maior Tetrapodomorpha descrito no Estado de São Paulo (CHAHUD \& PETRI 2010a), com as mesmas proporções, em tamanho, atribuídas à Temnospondyli de grande porte do Eopermiano da Bacia do Parnaíba, Prionosuchus plummeri, e do Neopermiano da Bacia do Paraná, Bageherpeton longignathus e Australerpeton cosgriffi (PRICE 1948, COX\&HUTCHINSON 1991, BARBERENA 1998, BARBERENA \& DIAS 1998, DIAS \& BARBERENA 2001, DIAS \& SCHULTZ 2003, VEGA-DIAS et al. 2008).
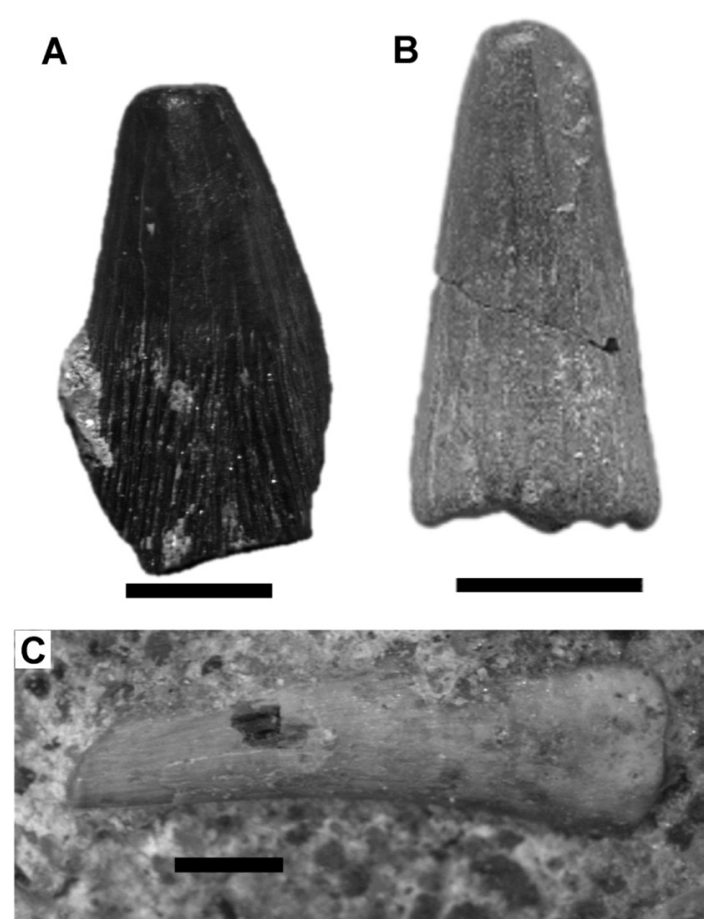

FIGURA 3 - Fósseis de Tetrapodomorpha da base arenosa do Membro Taquaral da Formação Irati na região de Rio Claro. A) Possível dente de Osteolepimorpha (GP/2E-6309) (CHAHUD \& PETRI 2009b). Escala $2 \mathrm{~mm}$. B) Dente labirintodonte de grande porte (GP/2T-202) (CHAHUD \& PETRI 2010a). Escala $10 \mathrm{~mm}$. C) Osso apendicular (GP/2E6457) (CHAHUD \& PETRI 2009b). Escala $1 \mathrm{~mm}$.

\section{CONSIDERAÇÕES PALEOMBIENTAIS}

Os Coelacanthimorpha e Tetrapodomorpha habitaram os mais variados ambientes e por isso as interpretações paleoambientais são muito complexas.

Atualmente, os Coelacanthimorpha são ótimos indicadores de ambiente marinho profundo, contudo, durante o Paleozoico habitaram águas doces e salgadas, não sendo bons indicadores paleoambientais.

Os Osteolepimorpha durante muito tempo foram bons indicadores de água doce e ambiente continental. No entanto, SWARTZ (2012) relatou a presença do osteolepiforme Tinirau clackae do Devoniano de Nevada, encontrado semi-articulado em camadas calcárias associadas a conularídeos, invertebrados tipicamente marinhos. Tal estudo comprova que o grupo não habitou apenas rios e lagos, mas também oceanos.

Ambientes salinos foram sugeridos para algumas espécies de Temnospondyli e tetrápodes, no entanto, algumas dessas interpretações podem estar equivocadas por desconsiderar aspectos bioestratigráficos, sedimentológicos e tafonômicos. O paleoambiente dos Tupilakosauridae, presentes no Permo-triássico da Europa, América do Norte, África do Sul e Groenlândia, foi discutido com base nos depósitos marinhos da Groenlândia. Porém, OCHEV \& SURKOV (2000) ressaltaram problemas estratigráficos e tafonômicos com esses fósseis. Outros Temnospondyli e até amniotas foram encontrados em ambientes salinos por transporte ou como carcaça e fragmentos de ossos.

Um crânio bem preservado, atribuído a Iberospondylus (Carbonífero, Bacia de Puertollano, Espanha), constitui um importante exemplo dessas controvérsias. Tal fóssil foi determinado como eurialino por LAURIN \& SOLER-GIJÓN (2001, 2006), baseado em análises geológicas da Bacia de Puertollano. Os autores utilizaram Chondrichthyes e microfósseis encontrados em água doce e salina para justificar o ambiente salino, desconsiderando: a total ausência de fósseis marinhos, uma camada de carvão associada, a presença de fósseis de água doce (conchostráceos, SOLERGIJÓN 1997a, b) e também a possibilidade do fóssil ter sido transportado como carcaça para a bacia.

Não se descarta a existência de tetrápodes basais em ambientes salinos (lagunares ou marinhos), contudo, é necessário um estudo aprofundado da unidade geológica, incluindo dados sedimentológicos e bioestratigráficos. 
Devido à característica cosmopolita dos Coelacanthimorpha no Paleozóico, os fósseis não foram importantes para interpretações paleoambientais na Bacia do Paraná. Contudo, os Tetrapodomorpha encontrados na base arenosa do Membro Taquaral foram considerados como indicadores de influência continental nos depósitos (CHAHUD 2007). Esses Tetrapodomorpha foram encontrados associados à Chondrichthyes de água doce (CHAHUD \& PETRI 2009b) e de águas salinas (RAGONHA 1978; CHAHUD et al. 2010; CHAHUD \& PETRI 2010b, 2012) no Membro Taquaral; desse modo, sua interpretação paleoambiental foi baseada em análise tafonômica e sedimentológica (CHAHUD 2007, 2011).

\section{CONSIDERAÇÕES FINAIS}

Escamas de Coelacanthimorpha foram observadas apenas na Formação Tatuí, enquanto no Membro Taquaral da Formação Irati também foram encontrados Tetrapodomorpha de tamanhos variados na base arenosa e escamas de celacantos na fácies folhelho síltico.

A presença de Coelacanthimorpha na Formação Tatuí e no Membro Taquaral comprovam que este grupo esteve presente em todo o Permiano da Bacia do Paraná, uma vez que também foram identificados em unidades subjacentes e sobrejacentes.

Os dentes labirintodontes identificados na base arenosa do Membro Taquaral do Estado de São Paulo podem ser atribuídos a diversos Tetrapodomorpha, Osteolepimorpha ou tetrápodes basais.

Apesar dos Coelacanthimorpha atualmente serem indicadores de ambiente marinho profundo, durante o Paleozoico, eles eram encontrados em ambientes de salinidade variável e também em água doce. Dessa forma, a interpretação paleoambiental destes peixes deve levar em consideração outras evidências, baseadas na tafonomia, sedimentologia e bioestratigrafia. O mesmo se aplica aos Tetrapodomorpha.

\section{AGRADECIMENTOS}

Os autores expressam seu agradecimento ao Conselho Nacional de Desenvolvimento Científico e Tecnológico $(\mathrm{CNPq})$, aos professores Thomas R. Fairchild e Marcello G. Simões pelo incentivo à realização deste trabalho e ao Departamento de Geologia Sedimentar e Ambiental do Instituto de Geociências da Universidade de São Paulo, onde foi realizada a pesquisa.

\section{REFERÊNCIAS BIBLIOGRÁFICAS}

ABREU, N.C.G.; DIAS-DA-SILVA, S. 2006. Proposta icnográfica para anfíbios fósseis: uma ferramenta em sistemática filogenética. GAEA, 2(1): 3-8.

AHLBERG, P.E.; JOHANSON, Z. 1998. Osteolepiforms and the ancestry of tetrapods. Nature, 395: 792-794.

ASSINE, M.L.; ZACHARIAS, A.A.; PERINOTTO, J.A.J. 2003 Paleocorrentes, paleogeografia e seqüências deposicionais da Formação Tatuí, centro-leste do Estado de São Paulo. Revista Brasileira de Geociencias, 33(1): 33-40.

BARBERENA, M.C. 1972. South America Late Paleozoic Tetrapods. Anais da Academia Brasileira de Ciências, 44 (suplemento): 67-75.

BARBERENA, M.C. 1998. Australerpeton cosgriffi n.g., n.sp. a Late Permian rhinesuchoid amphibian from Brazil. Anais da Academia Brasileira de Ciencias, (70)1: 125-147.

BARBERENA, M.C.; DIAS, E.V. 1998. On the presence of a short - snouted rhinesuchoid amphibian in the Rio do Rastro Formation (Late Permian of Paraná Basin, Brazil). Anais da Academia Brasileira de Ciencias, 70 (3): 465-467.

BARCELLOS, M.T. 1975. Estudo de escamas e dentes de peixes da Fácies Budó, Sub-grupo Itararé, RGS. Boletim Paranaense de Geociências, 32: 3-65.

BRYANT. W. L. 1929. Fossil fish remains from the Permian tillite of Brazil. Bulletin Geological Society American, 40: 424-426.

CARVALHO, M.S.S., MAISEY, J.G. 2008. New occurrence of Mawsonia (Sarcopterygii: Actinistia) from the Early Cretaceous of the Sanfranciscana Basin, Minas Gerais, southeastern Brazil. In: L. Cavin, A. Longbotton, M. Richter (eds.) Fishes and the Break-up of Pangaea. Geological Society, Special Publications 295, London, p. 109-144.

CHAHUD, A. 2007. Paleontologia de vertebrados da transição entre os grupos Tubarão e Passa Dois no Centro-Leste do Estado de São Paulo. Instituto de Geociências, Universidade de 
São Paulo, São Paulo, Dissertação de Mestrado, $172 \mathrm{p}$.

CHAHUD, A. 2011. Geologia e paleontologia das formações Tatuí e Irati no centro-leste do Estado de São Paulo. Instituto de Geociências, Universidade de São Paulo, São Paulo, Tese de Doutorado, 299 p.

CHAHUD, A.; PETRI, S. 2008a Chondrichthyes no Membro Taquaral, base da Formação Itati, no centro-leste do Estado de São Paulo, Brasil. Revista de Geologia, 21: 169-179.

CHAHUD, A.; PETRI, S. 2008b. Registro de paleoniscóides na base do Membro Taquaral, Formação Irati, Permiano da Bacia do Paraná. Revista do Instituto Geológico, 29(1/2): $33-40$.

CHAHUD, A.; PETRI, S. 2009a. Novos Xenacanthidae (Chondrichthyes, Elasmobranchii) da base do Membro Taquaral, Formação Irati, Permiano da Bacia do Paraná. Revista do Instituto Geológico, 30(1/2): 19-24.

CHAHUD, A.; PETRI, S. 2009b. Sarcopterygii do Topo da Formação Tatuí e Base da Formação Irati, Permiano, São Paulo, Brasil. Revista UnG Geociências, 8: 39-46.

CHAHUD, A.; PETRI, S. 2010a. Anfíbio e Paleonisciformes da Porção Basal do Membro Taquaral, Formação Irati (Permiano), Estado de São Paulo, Brasil. Geologia USP, Série Cientifica, 10(1): 29-37.

CHAHUD, A.; PETRI, S. 2010b. O tubarão Taquaralodus albuquerquei (Silva Santos, 1946) do Membro Taquaral (Permiano, Formação Irati) no Estado de São Paulo. Acta Biologica Paranaense, 39: 1-17.

CHAHUD, A.; PETRI, S. 2012. Levantamento dos cladodontes sul-americanos e novos espécimes do Membro Taquaral (Formação Irati, Permiano), Bacia do Paraná. Boletim Paranaense de Geociências, 66-67: 23-29.

CHAHUD, A.; PETRI, S. 2013a. Paleontology of Taquaral Member silty shale in the State of São Paulo. Brazilian Journal of Geology. 43: 117-123.

CHAHUD, A.; PETRI, S. 2013b. The silty shale Taquaral Member of the early Permian
Irati Formation (Paraná Basin, Brazil). Paleontology and paleoenvironments. Swiss Journal of Palaeontology. In press.

CHAHUD, A.; FAIRCHILD, T.R.; PETRI, S. 2010. Chondrichthyans from the base of the Irati Formation Permian, Parana Basin), São Paulo, Brazil. Gondwana Research, 18: 528537.

CHAHUD, A.; PACHECO, M.L.A.F.; MEIRA, F.E.; ROMERO, G.R.; PETRI, S. 2012. Paleontology and depositional environments of the Tatuí and Irati formations (Permian) in the Ponte Nova Farm, Ipeúna, state of São Paulo. Revista Brasileira de Geociências, 42(1): 198-212.

CISNEROS, J.C.; ABDALA, F.; MALABARBA, M.C. 2005. Pareiasaurids from the Riodo Rasto Formation, Southern Brazil: Bioestratigraphic Implications for Permian Faunas of the Paraná Basin. Revista Brasileira de Paleontologia, 8(1): 13-24.

COX, C.B.; HUTCHINSON, P. 1991. Fishes and amphibians from the Late Permian Pedra do Fogo Formation of Northern Brazil. Palaeontology, 34(3): 561-573.

DIAS, E.V. 2000. Anfíbios. In: I.S. Carvalho. (ed.) Paleontologia. Rio de Janeiro, Editora Interciência, p. 525-541.

DIAS, E.V.; BARBERENA, M.C.A 2001. Temnospondyl amphibian from the Rio do Rasto Formation, Upper Permian of Southern Brazil. Anais da Academia Brasileira de Ciências, 73(1): 135-143.

DIAS, E.V.; RICHTER, M. 2002. On the squamation of Australerpeton cosgriffi, Barberena, a temnospondyl amphibian from the Upper Permian of Brazil. Anais da Academia Brasileira de Ciências, 74(3): 477-490.

DIAS, E.V.; SCHULTZ, C.L. 2003. The First Paleozoic Temnospondyl Postcranial Skeleton from South America. Revista Brasileira de Paleontologia, 6: 29-42.

ELTINK, E.; DIAS, E.V. 2012. Temnospôndilos do Brasil: uma breve revisão e aspectos paleobiogeográficos. In: V. Gallo, H.M.A. Silva, P.M. Brito, F.J. Figueiredo (eds.) Paleontolo- 
gia de Vertebrados: Relações entre América do Sul e África. Rio de Janeiro, Interciência, p. 69-98.

FÚLFARO, V.J.; STEVAUX, J.C.; SOUZA FILHO, E.E.; BARCELOS, J.H. 1984. A Formação Tatuí $(\mathrm{P})$ no Estado de São Paulo. In: SBG, CONGRESSO BRASILEIRO DE GEOLOGIA, 23, Rio de Janeiro, Anais, 2: 711-723.

HACHIRO, J. 1996. O Subgrupo Irati (Neopermiano) da Bacia do Paraná. Instituto de Geociências, Universidade de São Paulo, São Paulo, Tese de Doutorado, 196 p.

LAURIN， M.; SOLER-GIJÓN. R. 2001. The oldest stegocephalian from the Iberian Peninsula: evidence that temnospondyls were euryhaline. Comptes Rendus de l'Académie des Sciences de Paris, Sciences de la Vie, 324: 495-501.

LAURIN, M.; SOLER-GIJÓN. R. 2006. The oldest known stegocephalian (Sarcopterygii: Temnospondyli) from Spain. Journal of Vertebrate Paleontology, 26(2): 284-299

MAISEY, J.G. 1991. Santana Fossils. An Ilustrated Atlas. T.F.H., United States of America, 459 p.

MARANHÃO, M.S.A.S. 1995. Fósseis das Formações Corumbataí e Estrada Nova do Estado de São Paulo: Subsídios ao conhecimento Paleontológico e Bioestratigráfico. Instituto de Geociências, Universidade de São Paulo, Tese de Doutorado, 2 volumes, $79 \mathrm{p}$.

MEZZALIRA, S.; MARTINS-NETO, R.G. 1992. Novos crustáceos paleozoicos do Estado de São Paulo com descrição de novos taxa. Acta Geológica Leopoldensia, Estudos Tecnológi$\cos , 15(36): 49-65$.

OCHEV, V.G.; SURKOV, M.V. 2000. The history of excavation of Permo-Triassic vertebrates from Eastern Europe. In: M.J. Benton, M.A. Shishkin, D.M. Unwin, E.N. Kurochkin (eds.) The Age of Dinosaurs in Russia and Mongolia. Cambridge, Cambridge University Press,. 1-16.

PRICE, L.I. 1948. Um anfíbio labirintodonte da Formação Pedra do Fogo, Estado do Maranhão. Boletim do DNPM/DGM, 124: 7-33.
PUTZER, H. 1954. Divisão da Formação Palermo no sul de Santa Catarina e tentativa de interpretação genética. Boletim da Sociedade Brasileira de Geologia, 3(1): 1-28.

RAGONHA, E.W. 1978. Chondrichthyes do Membro Taquaral (Formação Irati) no Estado de São Paulo. Instituto de Geociências, Universidade de São Paulo, São Paulo, Dissertação de Mestrado, $65 \mathrm{p}$.

RICHTER, M. 1985. Situação da Pesquisa Paleoictiológica no Paleozoico Brasileiro. In: Coletânea de Trabalhos Paleontológicos. DNPM, Série Geologia, Seção de Paleontologia e Estratigrafia, Brasília, 2 (27): 105-110.

RICHTER, M.; PICCOLI, A.M.M.; LIMA, M.C.S. 1985. Variação morfológica de restos de paleoniscídeos (Pisces) no Permiano da Bacia do Paraná. In: Coletânea de Trabalhos Paleontológicos. DNPM, Série Geologia. Seção de Paleontologia e Estratigrafia. Brasília. 2(27): 111-122.

SILVA SANTOS, R. 1991. Palaeonisciformes da Estado de São Paulo. In: SBP, CONGRESSO BRASILEIRO DE PALEONTOLOGIA, 12, São Paulo, Resumos, p. 11.

SOLER-GIJÓN, R. 1997a. Euselachian sharks from the Late Carboniferous of the Puertollano Basin, Spain: Biostratigraphic and palaeoenvironmental implications. Modern Geology, 21: 137-169.

SOLER-GIJÓN, R. 1997b. New discoveries of xenacanth sharks from the Late Carboniferous of Spain (Puertollano Basin) and Early Permian of Germany (Saar-Nahe Basin): Implications for the phylogeny of xenacanthiform and anacanthous sharks. Neues Jahrbuch fuer Geologie und Palaeontologie Abhandlungen, 205(1): 1-31.

SWARTZ, B. 2012. A Marine Stem-Tetrapod from the Devonian of Western North America. Plos one, 7(3): 1-11.

VEGA-DIAS, C.; ENNES-SILVA, R.A.; DIAS, E.V.; CHIMENTÃO, F.G. 2008. Novos espécimes de tetrápodes fósseis da Formação Rio do Rasto, Permiano da Bacia do Paraná, Brasil. In: SIMPÓSIO BRASILEIRO DE PALEONTOLOGIA DE VERTEBRADOS, 
6, Ribeirão Preto, Paleontologia em Destaque - Boletim da Sociedade Brasileira de Paleontologia, 1: 202-203.

WÜRDIG-MACIEL, N.L. 1975. Ichtiodontes e ichtiodorulitos (Pisces) da Formação Estrada
Nova e sua aplicação na estratigrafia do Grupo Passa Dois. Pesquisas, 5: 7-165.

YABUMOTO, Y. 2008. Anew Mesozoic coelacanth from Brazil (Sarcopterygii, Actinistia). Paleontological Research, 12: 329-343.

Endereço dos autores:

Artur Chahud e Setembrino Petri - Departamento de Geologia Sedimentar e Ambiental, Instituto de Geociências, Universidade de São Paulo, Rua do Lago, 562, Cidade Universitária, CEP 05508-900, São Paulo, SP, Brasil.E-mails: arturchahud@yahoo.com, spetri@usp.br

Artigo submetido em 6 de dezembro de 2012, aceito em 7 de outubro de 2013. 